\title{
Classical and quantum completeness for the Schrödinger op erators on non-compact manifolds
}

\author{
Mikhail Shubin
}

We provide a shorter and more transparent proof of a result by I. Oleinik [25, 26, 27]. It gives a sufficient condition of the essential self-adjointness of a Schrödinger operator on a non-compact Riemannian manifold with a locally bounded potential in terms of the completeness of the dynamics for a related classical system. The simplification of the proof given by I. Oleinik is achieved by an explicit use of the Lipschitz analysis on the Riemannian manifold and also by additional geometrization arguments which include a use of a metric which is conformal to the original one with a factor depending on the minorant of the potential.

\section{Introduction}

Let $(M, g)$ be a Riemannian manifold (i.e. $M$ is a $C^{\infty}$-manifold, $g=\left(g_{i j}\right)$ is a Riemannian metric on $M), \operatorname{dim} M=n$. We will always assume that $M$ is connected. Let $\Delta$ denote the Laplace-Beltrami operator on scalar functions on $M$ i.e.

$$
\Delta u=\frac{1}{\sqrt{g}} \frac{\partial}{\partial x^{i}}\left(\sqrt{g} g^{i j} \frac{\partial u}{\partial x^{j}}\right)
$$

where $x^{1}, \ldots, x^{n}$ are local coordinates, $\left(g^{i j}\right)$ is the inverse matrix to $g_{i j}, g=$ $\operatorname{det}\left(g_{i j}\right)$ and we use the usual summation convention.

The main object of our study will be the Schrödinger operator

$$
H=-\Delta+V(x)
$$

where the potential $V=V(x)$ is a real-valued measurable function which is in $L_{l o c}^{\infty}$ i.e. $V$ is locally bounded.

We will discuss conditions which guarantee that $H$ is essentially self-adjoint in the Hilbert space $L^{2}(M)=L^{2}(M, d \mu)$, where $d \mu=\sqrt{g} d x^{1} \ldots d x^{n}$ is the Riemannian volume element. This means that the closure of $H$ from the original domain $C_{c}^{\infty}(M)$ is a self-adjoint operator. (Here $C_{c}^{\infty}(M)$ is the set of all $C^{\infty}$ functions with compact support on $M$.)

The importance of the essential self-adjointness of $H$ becomes clear if we turn to the quantum mechanics and try to use the differential expression (1.1) to produce a quantum observable (a Hamiltonian) associated with this expression: a selfadjoint operator in $L^{2}(M)$ which extends $\left.H\right|_{C_{c}^{\infty}(M)}$. Such an extension always exists but essential self-adjointness means that this extension is unique. It is easy 
to see that this uniqueness is equivalent to the uniqueness of the solution of the following Cauchy problem for the evolutionary Schrödinger equation:

(1.2) $\frac{1}{i} \frac{\partial \psi(t)}{\partial t}=H \psi(t), \psi(0)=\psi_{0} \in C_{c}^{\infty}(M), \psi(t) \in L^{2}(M)$ for all $t \in \mathbb{R}$

(See e.g. [1], Ch.VI, Sect.1.7.) Here $H$ is applied to $\psi$ in the sense of distributions and the derivative in $t$ is taken in the norm sense.

In case when this uniqueness holds, it is natural to say that we have quantum completeness for the corresponding quantum system. (If the completeness does not hold, we need some extra data to construct a Hamiltonian, e.g. boundary conditions etc.)

Let us also consider the corresponding classical system: the Hamiltonian system with the Hamiltonian

$$
h(p, x)=|p|^{2}+V(x)
$$

in the cotangent bundle $T^{*} M$ (with the standard symplectic structure). Here $p$ is considered as a cotangent vector at the point $x \in M,|p|$ means the length of $p$ with respect to the metric induced by $g$ on $T^{*} M$. In local coordinates $\left(x^{1}, \ldots, x^{n}\right)$ we have

$$
|p|^{2}=g^{i j}(x) p_{i} p_{j}, \text { where } p=p_{j} d x^{j} \in T_{x}^{*} M .
$$

In the coordinates $\left(x^{1}, \ldots, x^{n}, p_{1}, \ldots, p_{n}\right)$ the hamiltonian system has the form

$$
\frac{d x^{i}}{d t}=\frac{\partial h}{\partial p_{i}}, \frac{d p_{i}}{d t}=-\frac{\partial h}{\partial x^{i}}, i=1, \ldots, n
$$

Let us assume for a moment that $V \in C^{2}(M)$, so the local Hamiltonian flow associated with the classical Hamiltonian (1.3) is well defined. Let us say that the system is classically complete if all the hamiltonian trajectories, i.e. solutions of (1.4), with arbitrary initial conditions are defined for all values of $t$. Usually it is more natural to require that they are defined for almost all initial conditions (in the phase space $T^{*} M$ ), but this distinction will not play any role in our considerations, though it is relevant if we want to treat potentials with local singularities (e.g. Coulomb type potentials).

We refer to Reed and Simon [30] for a more detailed discussion about classical and quantum completeness.

In the future we will assume that

$$
V(x) \geq-Q(x) \text { for all } x \in M
$$

where $Q$ is a real-valued function which is positive and somewhat more regular than $V$ itself.

For any $x, y \in M$ denote by $d_{g}(x, y)$ the distance between $x$ and $y$ induced by the Riemannian metric $g$.

Now we can formulate the main result: 
Theorem 1.1. (I. Oleinik [26]) Assume that $V$ satisfies (1.5) where $Q(x) \geq 1$ for all $x \in M$ and the following conditions are satisfied:

(a) The function $Q^{-1 / 2}$ is globally Lipschitz i.e.

$$
\begin{aligned}
\left|Q^{-1 / 2}(x)-Q^{-1 / 2}(y)\right| & \leq C d_{g}(x, y), x, y \in M, \\
\int^{\infty} Q^{-1 / 2} d s & =\infty,
\end{aligned}
$$

where the integral is taken along any parametrized curve (with a parameter $t \in$ $[a, \infty)$ ), such that it goes out to infinity (i.e. leaves any compact $K \subset M$ starting at some value of the parameter $t)$, ds means the arc length element associated with the given metric $g$.

Then the operator $H$ given by (1.1) is essentially self-adjoint.

Remark 1.2. The requirement $(b)$ is related to the classical completeness of the system with the Hamiltonian $|p|^{2}-Q(x)$ if we additionally assume that $Q \in$ $C^{2}(M)$. To illustrate this assume for simplicity that $M=\mathbb{R}^{n}$ and the metric $g$ is the standard flat metric on $\mathbb{R}^{n}$. Now assume that $(b)$ is satisfied. Then along the classical trajectory of the Hamiltonian $|p|^{2}-Q(x)$ we have

$$
|p|^{2}-Q(x)=E=\text { const. }
$$

It follows that

$$
d t=\frac{d s}{|\dot{x}|}=\frac{d s}{2|p|}=\frac{d s}{2 \sqrt{E+Q(x)}},
$$

hence the classical completeness for the Hamiltonian $\left|p^{2}\right|-Q(x)$ follows from the condition $(b)$.

Remark 1.3. If we assume that $Q \in C^{2}(M)$ then the condition $(b)$ is equivalent to the geodesic completeness of the Riemannian metric $\tilde{g}$ given by $\tilde{g}_{i j}=Q^{-1} g_{i j}$ (so $\tilde{g}$ is conformal to the original metric $g$ ).

Note also that $(b)$ implies that the original metric $g$ is also complete because $Q \geq 1$.

Remark 1.4. The requirement (a) in the theorem does not impose any serious restrictions on the growth of $Q$ at infinity, but rather restricts oscillations of $Q$. Indeed, we can equivalently rewrite (a) in the form of the following estimate:

$$
|d Q| \leq 2 C Q^{3 / 2},
$$

where $|d Q|$ means the length of the cotangent vector $d Q$ as above. Arbitrary tower of exponents

$$
e^{r}, e^{e^{r}}, e^{e^{e^{r}}} \ldots,
$$

satisfies this estimate. (Here $r=r(x)=d_{g}\left(x, x_{0}\right)$ with a fixed $x_{0} \in M$.)

Imposing appropriate conditions on $V$ sometimes leads to the equivalence of the conditions of classical and quantum completeness. An example of such situation 
was provided by A. Wintner [41] in case $n=1$, with the restrictions which mean that the derivatives of $V$ are small compared with $V$ itself. However some conditions are indeed necessary even in case $n=1$. This was shown by J. Rauch and M. Reed [29] who refer to unpublished lectures of E. Nelson. Examples given in [29] show that the classical and quantum completeness conditions are independent if no additional restrictions on $V$ are imposed.

Remark 1.5. Theorem 1.1 was extended to the Laplacian on forms by M.Braverman [3].

\section{Preliminaries on the Lipschitz analysis on a Riemannian manifold}

Let $(M, g)$ be a Riemannian manifold. A function $f: M \rightarrow \mathbb{R}$ is called a Lipschitz function with a Lipschitz constant $C$ if

$$
\left|f(x)-f\left(x^{\prime}\right)\right| \leq C d_{g}\left(x, x^{\prime}\right), \quad x, x^{\prime} \in M .
$$

It is well known that in this case $f$ is differentiable almost everywhere and

$$
|d f| \leq C
$$

with the same constant $C$. Here $|d f|$ means the length of the cotangent vector $d f$ in the metric associated with $g$. The corresponding differential $d f$, as well as the partial derivatives of the first order, coincide with the distributional derivatives. Vice versa if $d f \in L^{\infty}(M)$, for the distributional differential $d f=\left(\partial f / \partial x^{j}\right) d x^{j}$, then $f$ can be modified on a set of measure 0 so that it becomes a Lipschitz function.

The estimate (2.2) can be also rewritten in the form

$$
|\nabla f| \leq C
$$

(again with the same constant $C$ ), where $\nabla f$ means the gradient of $f$ associated with $g$, i.e. the vector field which corresponds to $d g$ and is given in local coordinates as

$$
\nabla f=g^{i j} \frac{\partial f}{\partial x^{i}} \frac{\partial}{\partial x^{j}} .
$$

In local form (in open subsets of $\mathbb{R}^{n}$ ) these facts are discussed e.g. in the book of V. Mazya [23], Sect.1.1. The correspondence between constants in (2.1), (2.2) and (2.3) is straightforward.

The Lipschitz vector fields, differential forms etc. are defined in an obvious way

We will need the Stokes formula, or rather the divergence formula for Lipschitz vector fields $v$ on $M$ in the following simplest form: 
Proposition 2.1. Let $v=v(x)$ be a Lipschitz vector field with a compact support on $M$. Then

$$
\int_{M} \operatorname{div} v d \mu=0
$$

Here div $v$ in local coordinates is given as

$$
\operatorname{div} v=\frac{1}{\sqrt{g}} \frac{\partial}{\partial x^{i}}\left(\sqrt{g} v^{i}\right), \quad v=v^{i} \frac{\partial}{\partial x^{i}},
$$

and $d \mu$ is the Riemannian volume element associated with $g$.

The proof of the Proposition can be easily reduced to the case when $v$ is supported in a domain of local coordinates. After that we can use mollification (regularization) of $v$ to approximate $v$ by smooth vector fields. A more general statement can be found in [23], Sect. 6.2.

\section{Proof of the main theorem}

Let $H_{\min }$ and $H_{\max }$ be the minimal and maximal operators associated with the differential expression (1.1) in $L^{2}(M)$. Here $H_{\min }$ is the closure of $H$ in $L^{2}(M)$ from the initial domain $C_{c}^{\infty}(M), H_{\max }=H_{\min }^{*}$ (the adjoint operator to $H_{\min }$ in $\left.L^{2}(M)\right)$. Clearly

$$
\operatorname{Dom}\left(H_{m a x}\right)=\left\{u \in L^{2}(M) \mid H u \in L^{2}(M)\right\},
$$

where $H u$ is understood in the sense of distributions.

It follows from the standard functional analysis arguments (see. e.g. [2], Appendix 1), that the essential self-adjointness of $H$ is equivalent to the symmetry of $H_{\max }$ which means that

$$
\left(H_{\max } u, v\right)=\left(u, H_{\max } v\right), \quad u, v \in \operatorname{Dom}\left(H_{\max }\right) .
$$

To establish the symmetry of $H_{\max }$ we need some information about Dom $\left(H_{\max }\right)$. This information is provided by the following

Lemma 3.1. If $u \in \operatorname{Dom}\left(H_{\max }\right)$, then

$$
\int_{M} Q^{-1}|\nabla u|^{2} d \mu \leq 2\left[\left(8 C^{2}+1\right)\|u\|^{2}+\|u\| \cdot\|H u\|\right]<\infty
$$

Here $\|\cdot\|$ means the norm in $L^{2}(M)$, and $C$ is the Lipschitz constant for $Q^{-1 / 2}$ from (1.6).

Proof. Let us choose a Lipschitz function $\phi: M \rightarrow \mathbb{R}$, such that $\phi$ has a compact support and

$$
0 \leq \phi \leq Q^{-1 / 2}
$$


Note that this implies that $\phi \leq 1$.

Let us estimate the quantity $I \geq 0$ where

$$
I^{2}=\int_{M} \phi^{2}|\nabla u|^{2} d \mu
$$

To this end note first that

$$
\begin{aligned}
\phi^{2}|\nabla u|^{2}= & \operatorname{div}\left(\phi^{2} u \nabla u\right)-2 \phi u \nabla \phi \cdot \nabla u-\phi^{2} u \cdot \Delta u= \\
& \operatorname{div}\left(\phi^{2} u \nabla u\right)-2 \phi u \nabla \phi \cdot \nabla u+\phi^{2} u \cdot H u-\phi^{2} u^{2} V \leq \\
& \operatorname{div}\left(\phi^{2} u \nabla u\right)-2 \phi u \nabla \phi \cdot \nabla u+\phi^{2} u \cdot H u+\phi^{2} u^{2} Q
\end{aligned}
$$

Here $\nabla \phi \cdot \nabla u$ means the scalar $g$-product of the tangent vectors $\nabla \phi$ and $\nabla u$. Let us integrate the inequality over $M$. By the Stokes formula (Proposition 2.1) the integral of the first term in the right hand side vanishes. Taking into account that $0 \leq \phi \leq 1$ and $\phi^{2} Q \leq 1$ due to (3.4), we can estimate the integral of the last two terms by $\|u\|(\|u\|+\|H u\|)$. Now denote by $\tilde{C}$ the Lipschitz constant of $\phi$, so that $|\nabla \phi| \leq \tilde{C}$. Then we obtain by the Cauchy-Schwarz inequality

$$
\begin{aligned}
& 2\left|\int_{M} \phi u \nabla \phi \cdot \nabla u\right|= \\
& 2\left|\int_{M}(u \nabla \phi) \cdot(\phi \nabla u)\right| \leq 2 \tilde{C} I|| u|| .
\end{aligned}
$$

Overall we obtain the inequality

$$
I^{2} \leq 2 \tilde{C} I\|u\|+\|u\|(\|u\|+\|H u\|) .
$$

Estimating

$$
2 \tilde{C} I\|u\| \leq \frac{1}{2} I^{2}+8 \tilde{C}^{2}\|u\|^{2},
$$

we arrive at the estimate

$$
I^{2} \leq 2\left[\left(8 \tilde{C}^{2}+1\right)\|u\|^{2}+\|u\| \cdot\|H u\|\right]
$$

Now it is easy to construct a sequence of Lipschitz functions $\phi_{k}, k=1,2, \ldots$, such that $\phi_{k}$ satisfies

$$
0 \leq \phi_{k} \leq Q^{-1 / 2}, \quad\left|\nabla \phi_{k}\right| \leq\left(C+\frac{1}{k}\right)
$$

(3.4) for any $k, \phi_{1} \leq \phi_{2} \leq \ldots$, and

$$
\lim _{k \rightarrow \infty} \phi_{k}(x)=Q^{-1 / 2}(x), \quad x \in M
$$


Indeed, take a function $\chi: \mathbb{R} \rightarrow \mathbb{R}$, such that $\chi \in C^{\infty}(\mathbb{R}), 0 \leq \chi \leq 1, \chi(t)=1$ if $t \leq 1, \chi(t)=0$ if $t \geq 3$, and $\left|\chi^{\prime}\right| \leq 1$. Then we can take

$$
\phi_{k}(x)=\chi\left(k^{-1} d_{g}\left(x, x_{0}\right)\right) Q^{-1 / 2}(x)
$$

where $x_{0} \in M$ is an arbitrary fixed point. The estimate (3.6) holds for $\phi_{k}$ with $\tilde{C}=C+\frac{1}{k}$. Taking the limit as $k \rightarrow \infty$, we obtain (3.3).

Proof of Theorem 1.1. Let us introduce the metric $\tilde{g}_{i j}=Q^{-1} g_{i j}$ and denote the corresponding distance function by $\tilde{d}$. This means that

$$
\tilde{d}(x, y)=\inf \left\{\int_{\gamma} Q^{-1 / 2} d s \mid \gamma:[0,1] \rightarrow M, \gamma(0)=x, \gamma(1)=y\right\}
$$

where $\gamma \in C^{\infty}$ and $d s$ means the element of the arc length of $\gamma$ associated with $g$. Denote also

$$
P(x)=\tilde{d}\left(x, x_{0}\right)
$$

where $x_{0} \in M$ is fixed. The completeness condition $(b)$ means exactly that

$$
P(x) \rightarrow \infty \text { as } x \rightarrow \infty,
$$

or, equivalently, that the set $\{x \mid P(x) \leq t\} \subset M$ is compact for any $t \in \mathbb{R}$.

Clearly, $|d P|_{\tilde{g}} \leq 1$, which can be rewritten as $|d P|_{g}^{2} \leq Q^{-1}$, or

$$
|\nabla P| \leq Q^{-1 / 2}
$$

(Here, as above, $\nabla P$ means the gradient with respect to the original metric $g$, and $|\nabla P|$ means the length of the tangent vector $\nabla P$ with respect to $g$.

Now for two real-valued functions $u, v \in \operatorname{Dom}\left(H_{\max }\right)$ consider the following integral:

$$
I_{t}=\int_{\{x \mid P(x) \leq t\}}\left(1-\frac{P(x)}{t}\right)(u \cdot H v-v \cdot H u) d \mu .
$$

By the dominated convergence theorem we obviously have

$$
I_{t} \rightarrow \int_{M}(u \cdot H v-v \cdot H u) d \mu=(u, H v)-(H u, v) \quad \text { as } t \rightarrow \infty
$$

(Here $(\cdot, \cdot)$ means the scalar product in $L^{2}(M)$.)

Now note that

$$
u \cdot H v-v \cdot H u=v \cdot \Delta u-u \cdot \Delta v=\operatorname{div}(v \nabla u-u \nabla v)
$$

and rewrite the integrand of $I_{t}$ as

$$
\operatorname{div}\left[\left(1-\frac{P(x)}{t}\right)(v \nabla u-u \nabla v)\right]+\frac{1}{t}(\nabla P)(v \nabla u-u \nabla v)
$$


The integral of the first term vanishes due to Proposition 2.1. Therefore using the Cauchy-Schwarz inequality we obtain

$$
\begin{aligned}
\left|I_{t}\right| & =\left|\frac{1}{t} \int_{\{x \mid P(x) \leq t\}}(\nabla P) \cdot(v \nabla u-u \nabla v) d \mu\right| \\
& =\left|\frac{1}{t} \int_{\{x \mid P(x) \leq t\}}\left(Q^{1 / 2} \nabla P\right) \cdot Q^{-1 / 2}(v \nabla u-u \nabla v) d \mu\right| \\
& \leq \frac{1}{t}\left(\|v\||| Q^{-1 / 2} \nabla u\|+\| u\left|\left\|\mid Q^{-1 / 2} \nabla v\right\|\right)\right.
\end{aligned}
$$

By Lemma 3.1 the right hand side is $O(1 / t)$, so $I_{t} \rightarrow 0$ as $t \rightarrow \infty$. Due to (3.10) this proves that $H_{\max }$ is symmetric i.e. (3.2) holds. This ends the proof of Theorem 1.1.

\section{Examples and further comments}

In this section we will provide several examples, further results and relevant bibliographical comments (by necessity incomplete).

We will start with some particular cases of Theorem 1.1 .

Theorem 4.1. (M. Gaffney [12]) Let $(M, g)$ be a complete Riemannian manifold. Then the Laplace-Beltrami operator $\Delta$ is essentially self-adjoint.

Proof. Take $Q(x) \equiv 1$ and use Theorem 1.1 .

Note that in fact the proof of Theorem 1.1 uses some elements of the Gaffney's proof.

Theorem 4.2. Let $(M, g)$ be a complete Riemannian manifold, $V \in L_{l o c}^{\infty}(M)$, and $V(x) \geq-C, x \in M$, with a constant $C$. Then the Schrödinger operator $H=-\Delta+V(x)$ is essentially self-adjoint.

In case when $M=\mathbb{R}^{n}$ (with the standard metric) this result is due to T. Carleman [5], and the Carleman proof is reproduced in the book of I.M. Glazman [14], Theorem 34 in Sect.3.

It is actually sufficient to require only that the operator $H_{\min }$ is semi-bounded below, as was suggested by I.M. Glazman and proved by A.Ya. Povzner [28]. Another proof was suggested by E. Wienholtz [40] and also reproduced in [14].

Though the completeness requirement looks natural in case of semi-bounded operators, sometimes it can be relaxed and incompletness may be compensated by a specific behavior of the potential (see e.g. A.G. Brusentsev [4] and also the references there). 
The following theorem in case $M=\mathbb{R}^{n}$ is due to D.B. Sears (see e.g. $[35,39,2]$ ), who followed an idea of an earlier paper by E.C. Titchmarsh.

Theorem 4.3. Let us fix $x_{0} \in M$ and denote $r=r(x)=d_{g}\left(x, x_{0}\right)$. Assume that $V(x) \geq-Q(r)$ where $Q(r) \geq 1$ for all $r \geq 0$,

$$
\int_{0}^{\infty} \frac{d r}{\sqrt{Q(r)}}=\infty
$$

and one of the following two conditions is satisfied:

(a) $Q^{-1 / 2}$ is globally Lipschitz, i.e.

$$
\left|Q^{-1 / 2}(r)-Q^{-1 / 2}\left(r^{\prime}\right)\right| \leq C\left|r-r^{\prime}\right|, \quad r, r^{\prime} \in[0, \infty)
$$

(b) $Q$ is continuous and monotone increasing.

Then the operator (1.1) is essentially self-adjoint.

Proof. Under condition $(a)$ this theorem clearly follows from Theorem 1.1.

Now assume that $(b)$ is satisfied. Then we can follow F.S. Rofe-Beketov [31] to reduce this to the case when in fact $(a)$ is satisfied. It is enough to construct a new function $\tilde{Q}$, such that $\tilde{Q}(r)>Q(r)$ for all $r \geq 0$ and $\tilde{Q}$ satisfies both (4.1) and (a). To this end we can define $\tilde{Q}(n)=Q(n+1), n=0,1,2, \ldots$, and then extend $\tilde{Q}^{-1 / 2}$ to the semi-axis $[0, \infty)$ by linear interpolation, i.e. take

$$
\tilde{Q}^{-1 / 2}(\alpha n+(1-\alpha)(n+1))=\alpha \tilde{Q}^{-1 / 2}(n)+(1-\alpha) \tilde{Q}^{-1 / 2}(n+1),
$$

where $0 \leq \alpha \leq 1, n=0,1, \ldots$. It is easy to see that $\tilde{Q}$ satisfies the desired conditions.

Remark 4.4. F.S. Rofe-Beketov [32] proved (in case $M=\mathbb{R}^{n}$ ) that the local inequality $V(x) \geq-Q(x)$ can be replaced by an operator inequality

$$
H \geq-\varepsilon \Delta-Q(x)
$$

with a constant $\varepsilon>0$. This allows in particular some potentials which are unbounded below. I. Oleinik [27] noticed that this result can be carried over to the case of manifolds as well.

Remark 4.5. F.S. Rofe-Beketov [31] noticed that if in Theorem 4.3 we have $Q(r)<\infty$ for all $r \geq 0$ and $Q$ satisfies (4.2), then we can always replace $Q$ by another function $Q_{1} \in C^{\infty}$ such that $Q_{1}$ also satisfies all the conditions (including (a) with a possibly bigger Lipschitz constant).

Indeed, it suffices to construct a globally Lipschitz $C^{\infty}$ function $Q_{1}:[0, \infty) \rightarrow$ $[1, \infty)$ so that $Q(r) / 2 \leq Q_{1}(r) \leq 2 Q(r)$ for all $r \geq 0$. To this end we can first 
mollify $Q^{-1 / 2}$ on each of the overlapping intervals $[0,4],[2,6],[6,10], \ldots$, by convolution with a positive smooth probability measure supported in a small neighborood of 0 . This neighborhood should depend on the chosen interval to insure the desired inequalities. Note that the convolution does not change the Lipschitz constant. Then we can use a partition of unity on $[0, \infty)$ such that it is subordinated to the covering of $[0, \infty)$ by the intervals above and consists of functions which have uniformly bounded derivatives of any fixed order (e.g. translations of an appropriately fixed $C^{\infty}$ function). Using such partition of unity to glue locally mollified function $Q^{-1 / 2}$ we arrive to the desired approximation $Q_{1}^{-1 / 2}$.

Remark 4.6. A more general Sears-type result was obtained by T. Ikebe and T. Kato [17] where magnetic Schrödinger operators in $\mathbb{R}^{n}$ with possibly locally singular potentials were considered. The allowed local singularities are most naturally described by the Stummel type conditions first introduced by F. Stummel [38]; see also E. Wienholtz [40], E. Nelson [24], K. Jörgens [19], G. Hellwig [16], T. Kato [20], B. Simon [37] and references there for other results on operators with singular potentials.

Remark 4.7. B.M. Levitan [22] gave a new proof of Theorem 4.3 (in case $M=\mathbb{R}^{n}$ with the flat metric). His proof uses the wave equation and the finite propagation speed argument. Similar arguments were later used by A.A. Chumak [7], P. Chernoff [6] and T. Kato [21] to prove essential self-adjointness in a somewhat different context. A.A. Chumak considered semi-bounded Schrödinger operators on complete Riemannian manifolds. P. Chernoff proves in particular the essential self-adjointness for the powers of such operators as well as Dirac operators, whereas T. Kato extends the arguments and results to the powers $H^{m}, m=1,2, \ldots$, (in $\mathbb{R}^{n}$ ) under the condition that $H \geq-a-b|x|^{2}$ with some constants $a, b$.

Note however that the self-adjointness of the powers of the Laplacian on a complete Riemannian manifold was first established by H.O. Cordes [8] without finite propagation speed argument. (See also the book [9] for a variety of results on essential self-adjointness of semi-bounded Schrödinger-type operators on manifolds and their powers.)

There are many results on self-adjointness of more general higher order operators - see e.g. M. Schechter [34] for operators in $\mathbb{R}^{n}$ (and also for similar $L^{p}$ results in $\mathbb{R}^{n}$ ) and also M. Shubin [36] for operators on manifolds of bounded geometry, as well as F.S. Rofe-Beketov [33] and references there.

Now we will formulate a result of I. Oleinik which shows that in fact it is sufficient to restrict the behavior of the potential $V$ only on some sequence of layers or shells which eventually surround all the points in $M$. From the classical point of view this is obvious because the classical completeness can be guaranteed if the classical particle escaping to infinity spends infinite time already inside the layers. The first result of this kind in case $n=1$ is due to P. Hartman [15], and further generalizations were obtained in one-dimensional case by R. Ismagilov [18] (higher 
order operators), and in case $M=\mathbb{R}^{n}$ by M.G. Gimadislamov [13], F.S. RofeBeketov [32], M.S.P. Eastham, W.D. Evans, J.B. McLeod [11] and A. Devinatz [10] (the last two references also include magnetic field terms).

Theorem 4.8. (I. Oleinik [27]) Let $\left\{\Omega_{k} \mid k=0,1, \ldots,\right\}$ be a sequence of open relatively compact subsets with smooth boundaries in $M, \overline{\Omega_{k}} \subset \Omega_{k+1}, \cup_{k} \Omega_{k}=M$. Denote $T_{k}=\Omega_{2 k+1} \backslash \overline{\Omega_{2 k}}$, and let $h_{k}$ be the minimal thickness of the layer $T_{k}$, i.e. $h_{k}=\operatorname{dist}_{g}\left(\Omega_{2 k}, M \backslash \Omega_{2 k+1}\right)$. Assume that

$$
V(x) \geq-C \gamma_{k}, x \in T_{k}, \quad k=0,1, \ldots,
$$

where $C>0, \gamma_{k} \geq 1$, and

$$
\sum_{k=0}^{\infty} \min \left\{h_{k}^{2}, h_{k} \gamma_{k}^{-1 / 2}\right\}=\infty
$$

Then the operator (1.1) is essentially self-adjoint.

Proof. Following F.S. Rofe-Beketov [32] and I. Oleinik [27] we will construct a minorant $Q$ for the potential $V$, so that the conditions $(a)$ and $(b)$ in Theorem 1.1 are satisfied.

We will start by constructing for any $k=0,1, \ldots$, a function $Q_{k} \geq 0$ on $M$ such that $Q_{k}=+\infty$ on $M \backslash T_{k}$, then assemble $Q^{-1 / 2}$ as a linear combination of the functions $Q_{k}^{-1 / 2}$.

Denote for any $x \in M$

$$
\delta_{2 k}(x)=\operatorname{dist}_{g}\left(x, \Omega_{2 k}\right), \quad \delta_{2 k+1}(x)=\operatorname{dist}_{g}\left(x, M \backslash \Omega_{2 k+1}\right), \quad k=0,1, \ldots
$$

For $p=2 k, 2 k+1$ introduce sets

$$
\Omega_{p}^{\prime}=\left\{x \mid \delta_{p}(x) \leq h_{k} / 4\right\}
$$

and functions $\delta_{p}^{\prime}: M \rightarrow[0, \infty)$,

$$
\delta_{p}^{\prime}(x)=\operatorname{dist}_{g}\left(x, M \backslash \Omega_{p}^{\prime}\right) .
$$

Now define

$$
Q_{k}^{-1 / 2}(x)=h_{k}^{-1}, \quad x \in M \backslash\left(\Omega_{2 k}^{\prime} \cup \Omega_{2 k+1}^{\prime}\right),
$$

and

$$
Q_{k}^{-1 / 2}(x)=h_{k}^{-1} \delta_{p}(x)\left(\delta_{p}(x)+\delta_{p}^{\prime}(x)\right)^{-1}, \quad x \in \Omega_{p}^{\prime},
$$

where $p=2 k$ or $2 k+1$. Clearly $0 \leq Q_{k}^{-1 / 2}(x) \leq h_{k}^{-1}$ on $M$ and $Q_{k}^{-1 / 2}(x)=0$ if $x \notin T_{k}$.

Let us evaluate the Lipschitz constant for $Q_{k}^{-1 / 2}$. To this end denote $f(s, t)=$ $s /(s+t)$, and observe that the absolute values of both partial derivatives of $f$ in 
$s$ and $t$ are bounded by $(s+t)^{-1}$ if $s, t \geq 0, s+t>0$. Also both $\delta_{p}$ and $\delta_{p}^{\prime}$ are Lipschitz with the Lipschitz constant 1 . Now note that it is easily follows from the triangle inequality that

$$
\delta_{p}(x)+\delta_{p}^{\prime}(x) \geq h_{k} / 4, \quad x \in M .
$$

Hence by the chain rule we see that

$$
\left|\nabla\left(Q_{k}^{-1 / 2}\right)\right| \leq 2 h_{k}^{-1} \cdot 4 h_{k}^{-1}=8 h_{k}^{-2} .
$$

Hence the Lipschitz constant of $Q_{k}^{-1 / 2}$ does not exceed $8 h_{k}^{-2}$.

Now let us define

$$
Q^{-1 / 2}(x)=\sum_{k=0}^{\infty} a_{k} Q_{k}^{-1 / 2},
$$

where we will adjust the coefficients $a_{k} \geq 0$ so that all the conditions are satisfied. Let us list these conditions turn by turn.

(a) We need the condition $V \geq-Q$ to be satisfied which will be guaranteed if $-C \gamma_{k} \geq-Q(x), x \in T_{k}$. This is equivalent to $Q_{k}^{-1 / 2} \leq\left(C \gamma_{k}\right)^{-1 / 2}, k=0,1, \ldots$, and will be guaranteed if $a_{k} h_{k}^{-1} \leq\left(C \gamma_{k}\right)^{-1 / 2}$ or

$$
a_{k} \leq C^{-1 / 2} h_{k} \gamma_{k}^{-1 / 2}
$$

(b) The Lipschitz constant of $Q^{-1 / 2}$ is evaluated by $8 \sup _{k}\left(a_{k} h_{k}^{-2}\right)$, so for $Q^{-1 / 2}$ to be Lipschitz it is sufficient to have

$$
a_{k} \leq C_{1} h_{k}^{2}
$$

with some constant $C_{1}>0$.

(c) At last we need the condition (b) of Theorem 1.1 to be satisfied. Note that the minimal thickness of the internal layer $T_{k}^{\prime}=M \backslash\left(\Omega_{2 k}^{\prime} \cup \Omega_{2 k+1}^{\prime}\right)$ is at least $h_{k} / 2$, and $Q^{-1 / 2}=a_{k} h_{k}^{-1}$ in $T_{k}^{\prime}$. It follows that the condition $(b)$ in Theorem 1.1 will be satisfied if we require

$$
\sum_{k=0}^{\infty} a_{k}=\infty .
$$

Now taking $C_{1}=C^{-1 / 2}$ we can choose

$$
a_{k}=C^{-1 / 2} \min \left\{h_{k}^{2}, h_{k} \gamma_{k}^{-1 / 2}\right\},
$$

so the conditions (4.6), (4.7) will be automatically satisfied. The condition (4.8) will be satisfied if we require the condition (4.5) to hold.

\section{Acknowledgements}

This work was partially supported by NSF grant DMS-9706038. I am also very grateful to Yu. Netrusov and F.S. Rofe-Beketov for useful discussions. 


\section{References}

[1] Yu.M. Berezanski, Expansions in eigenfunctions of self-adjoint operators, Amer. Math. Soc. Translation of Math. Monographs, Providence, RI, 1968

[2] F.A. Berezin, M.A. Shubin, The Schrödinger equation, Kluwer Academic Publishers, Dordrecht e.a., 1991

[3] M. Braverman, On self-adjointness of a Schrödinger operator on differential forms, Proc. Amer. Math. Soc., 126 (1998), 617-624

[4] A.G. Brusentsev, On essential self-adjointness of semi-bounded second order elliptic operators without completeness of the Riemannian manifold, Math. Physics, Analysis, Geometry (Kharkov), 2 (1995), no. 2, 152-167 (in Russian)

[5] T. Carleman, Sur la théorie mathématique de l'équation de Schrödinger, Ark. Mat. Astr. Fys., 24B, no. 11 (1934), 1-7

[6] P. Chernoff, Essential self-adjointness of powers of generators of hyperbolic equations, J. Funct. Analysis, 12 (1973), 401-414

[7] A.A. Chumak, Self-adjointness of the Beltrami-Laplace operator on a complete paracompact manifold without boundary, Ukrainian Math. Journal, 25 (1973), no. 6, 784791 (in Russian)

[8] H.O. Cordes, Self-adjointness of powers of elliptic operators on non-compact manifolds, Math. Annalen, 195 (1972), 257-272

[9] H.O. Cordes, Spectral theory of linear differential operators and comparison algebras, London Math. Soc., Lecture Notes Series, 76, Cambridge Univ. Press, 1987

[10] A. Devinatz, Essential self-adjointnessof Schrödinger-type operators, J. Funct. Analysis, 25 (1977), 58-69

[11] M.S.P. Eastham, W.D. Evans, J.B. McLeod The essential self-adjointness of Schrödinger type operators, Arch. Rat. Mech. Anal., 60 (1975/76), no. 2, 185-204

[12] M. Gaffney, A special Stokes's theorem for complete Riemannian manifolds, Ann. of Math., 60 (1954). 140-145

[13] M.G. Gimadislamov, Sufficient conditions of coincidence of minimal and maximal partial differential operators and discreteness of their spectrum, Math. Notes, 4, no. 3 (1968), 301-317 (in Russian)

[14] I.M. Glazman, Direct methods of qualitative spectral analysis of singular differential operators, Israel Program for Scientific Translation, Jerusalem, 1965

[15] P. Hartman, The number of $L^{2}$-solutions of $x^{\prime \prime}+q(t) x=0$, Amer. J. Math., 73 (1951), 635-645

[16] G. Hellwig, Differential operators of mathematical physics. An introduction. Addison-Wesley, 1964

[17] T. Ikebe, T. Kato, Uniqueness of the self-adjoint extension of singular elliptic differential operators, Arch. for Rat. Mech. and Anal., 9 (1962), 77-92

[18] R.S. Ismagilov, Conditions for self-adjointness of differential operators of higher order, Dokl. Akad. Nauk SSSR, 142 (1962), 1239-1242. English translation: Soviet Math. Doklady, 3 (1962), 279-283 
[19] K. Jörgens, Wesentliche Selbstadjungiertheit singulärer elliptischer Differentialoperatoren zweiter Ordnung in $C_{0}^{\infty}(G)$, Math. Scand., 15 (1964), 5-17

[20] T. Kato, Schrödinger operators with singular potentials, Israel J. Math., 13 (1972), $135-148$

[21] T. Kato, A remark to the preceding paper by Chernoff, J. Funct. Analysis, 12 (1973), 415-417

[22] B.M. Levitan, On a theorem by Titchmarsh and Sears, Uspekhi Matem. Nauk, 16, no.4 (1961), 175-178 (in Russian)

[23] V.G. Mazya, Sobolev spaces, Springer-Verlag, Berlin e.a., 1985

[24] E. Nelson, Feynman integrals and the Schrödinger operators, J. Math. Phys., 5 (1964), 332-343

[25] I.M. Oleinik, On the essential self-adjointness of the Schrödinger operator on a complete Riemannian manifold, Mathematical Notes, 54 (1993), 934-939

[26] I.M. Oleinik, On the connection of the classical and quantum mechanical completeness of a potential at infinity on complete Riemannian manifolds, Mathematical Notes, 55 (1994), 380-386

[27] I.M. Oleinik, On the essential self-adjointness of the Schrödinger-type operators on complete Riemannian manifolds, $\mathrm{PhD}$ thesis, Northeastern University, May 1997

[28] A.Ya. Povzner, On expansions of arbitrary functions in eigenfunctions of the operator $\Delta u+c u$, Matem. Sbornik, 32 (74), no. 1 (1953), 109-156 (in Russian)

[29] J. Rauch, M. Reed, Two examples illustrating the differences between classical and quantum mechanics, Commun. Math. Phys., 29 (1973), 105-111

[30] M. Reed, B. Simon, Methods of modern mathematical physics. II: Fourier analysis, self-adjointness. Academic Press, New York e.a., 1975

[31] F.S. Rofe-Beketov, On non-semibounded differential operators, Theory of Functions, Functional Analysis and Applications (Teoriya funktsii, funkts. analyz i ikh prilozh.), no. 2, Kharkov (1966), 178-184 (in Russian)

[32] F.S. Rofe-Beketov, Conditions for the self-adjointness of the Schrödinger operator, Mathematical Notes, 8 (1970), 888-894

[33] F.S. Rofe-Beketov, Self-adjointness of elliptic operators of higher order and energy estimates in $\mathbb{R}^{n}$, Theory of Functions, Functional Analysis and Applications (Teoriya funktsii, funkts. analyz i ikh prilozh.), no. 56, Kharkov (1991), 35-46 (in Russian)

[34] M. Schechter, Spectra of partial differential operators, North-Holland, 1971

[35] D.B. Sears, Note on the uniqueness of Green's functions associated with certain differential equations, Canad. J. Math., 2 (1950), 314-325

[36] M.A. Shubin, Spectral theory of elliptic operators on non-compact manifolds, Astérisque, 207 (1992), 35-108

[37] B. Simon, Essential self-adjointness of Schrödinger operators with positive potentials, Math. Annalen, 201 (1973), 211-220

[38] F. Stummel, Singuläre elliptische Differentialoperatoren in Hilbertschen Räumen, Math. Annalen, 132 (1956), 150-176 
[39] E.C. Titchmarsh, Eigenfunction expansions associated with second-order differential equations, Part II, Clarendon Press, Oxford,1958

[40] E. Wienholtz, Halbbeschränkte partielle Differentialoperatoren zweiter ordnung vom elliptischen Typus, Math. Ann., 135 (1958), 50-80

[41] A. Wintner, On the normalization of characteristic differentials in continuous spectra, Phys. Rev., 72 (1947), 516-517

Department of Mathematics, Northeastern University, Boston, MA 02115, USA

1991 Mathematics Subject Classification: Primary 35P05, 58G25; Secondary 47B25, 81Q10

Submitted: Date inserted by the Editor 